\title{
Artificial Neural Network based Lesion Segmentation of Brain MRI
}

\author{
Tapas Si \\ Dept. of ECE \\ National Institute of Technology Durgapur \\ West Bengal, India
}

\author{
Arunava De \\ Dept. of IT \\ Dr. B.C. Roy Engineering College \\ Duragpur, West Bengal, India
}

\author{
Anup Kumar Bhattacharjee \\ Dept. of ECE \\ National Institute of Technology Durgapur \\ West Bengal, India
}

\begin{abstract}
An Artificial Neural Network based segmentation method for lesion in brain is proposed. First, Magnetic Resonance Images (MRI) are denoised and intensity inhomogeneities are corrected in the preprocessing steps. Artificial neural network is used for training using gray levels and extracted statistical features from the training data with the labelled ground truth. The test images are segmented into lesion and healthy tissues using trained neural network. The connected component labelling algorithm is used to extract only lesion from the segmented images. The proposed method is applied on two MRI data set. The performance of the proposed method is compared with K-means algorithm. The proposed method performs better than K-means algorithm both qualitatively and quantitatively.
\end{abstract}

\section{General Terms}

Medical Image Processing, Machine Learning

\section{Keywords}

Brain, Magnetic Resonance Image, Lesion, Segmentation, Artificial Neural Network

\section{INTRODUCTION}

Multimodal MR Image (MRI) [1] segmentation is an important medical image analysis task. Lesion detection is a preliminary step in disease diagnosis. Magnetic Resonance Imaging is now an important tool for effective diagnosis, treatment and monitoring of the brain disease [2]. MR images of brain can have maximum seven objects [3] such as: (i) background, (ii) white matter (WM), (iii) gray matter (GM), (iv) Cerebrospinal fluid (CSF), (v) bone, (vi) scalp and (vii) lesion. Different modalities of brain MRI of the same patient such as: (a) T1-Weighted (b) T2-Weighted (c) Proton density ( $\rho$-weighted) (d) Fluid-attenuated inversion recovery (FLAIR) (e) diffusionweighted (DW) (f) Perfusion-weighted (PW) exist [1] . Machine Learning (ML) plays a key role in many radiology applications and it helps radiologists make intelligent decisions on CT, MRI, PET images and radiology reports [6]. Machine Learning techniques are used in developing computer aided detection or diagnosis (CAD) system [6, 7]. A. Mayer and H. Greenspan [8] proposed an adaptive mean-shift clustering algorithm to classify the brain voxels into white matter, gray matter and Cerebro-spinal fluid. T. Si et al. [9] proposed Grammatical Swarm (GS) based segmentation method for brain MRI in which GS based hardclustering technique is used to segment the lesion in brain. This method performs better than K-Means and Fuzzy c-means algorithm in lesion segmentation. T. Si et al. [10] proposed a hardclustering technique with Grammatical Swarm based-adaptable Particle Swarm Optimizer (PSO) in segmentation of MRI for lesion detection in brain. The proposed method performs better than PSO based clustering technique. T. Si et al. [11] proposed a entropy maximization based segmentation method using Grammatical Swarm for lesion detection in brain MRI.
Artificial Neural Network (ANN) [4], a machine learning tool, is now widely used in segmentation of brain MRI. J. Alirezaie et al. [3] proposed Back-Propagation neural network and Learning Vector Quantization (LVQ) neural network to segment the brain MR images into different objects. In this method, a multilayer perceptron (MLP) was trained with extracted features from Axial-T1, Axial-T2 and $\rho$ weighted MR images and tested with images that were not used in training. The well-known BackPropagation (BP) algorithm was used to train MLP. Similarly, LVQ neural network is also used to classify the different objects in MR images. E.S. A. E. Dahshan et al. [7] proposed a hybrid intelligent machine learning technique in which principle component analysis (PCA) was used for reducing the Wavelet features. Feed-forward multilayer neural network (FFNN) was used for classification for automatic detection of brain tumor.

M.S. Yang et al. [12] proposed a segmentation method for brain MR images using fuzzy-soft LVQ neural network. N. Zhang et al. [13] proposed tumor segmentation in brain MRI using Support Vector Machine (SVM) with feature selection in kernel space.

A. Ortiz et al. [14] proposed two unsupervised neural techniques for brain MR image segmentation. In first method, they used histogram to segment the whole volume using Self-Organizing Map (SOM). In the second method, the features are extracted from the images and Genetic Algorithm (GA) is used to select the suitable features. Finally, the SOM is used to classify the selected features into clusters with entropy gradient clustering method.

T. Song et al. [15] proposed a weighted probabilistic neural network (WPNN) for partial volume segmentation in brain MR image. Y. Zhang et al. [16] proposed a neural network based method to classify brain MR image as normal or abnormal. In this method, discrete wavelet transform (DWT) is used to extract the features from MR images and principle component analysis (PCA) is used to select the features. A back propagation neural network trained with scaled conjugate gradient (SCG) method is used to classify the MR images. Y. Zhang et al. [17] proposed a brain MR image classifier system using Kernel Support Vector Machine (KSVM). DWT is used to extract the features and PCA is used to select the features. The selected features are used as inputs in KSVM to classify the MR images. PSO algorithm is used to optimize the parameters of KSVM.

Based on the existing literatures survey, it is found that ANN is used in brain MRI in two ways: (i) segmentation of different tissues [3, 13, 14, 15] and (ii) classification of MRI slice into either normal or abnormal status [7, 16, 17]. The first approach classifies the MRI pixels into different class and abnormal tissues are detected manually if present. The objective of this work is to detect lesion in brain MRI without classifying the MR image into different tissue types (i.e WM, GM, CSF etc.). Therefore, a binary classifier is developed in this work using a Multi-Layer Perceptron (MLP) neural network. The brain tissues are classified into lesion and healthy tissue types using the proposed classifier. In the proposed method, MR images are denoised and intensity inhomogeneities are corrected in the preprocessing steps. A set of statistical features are extracted from the MR images. These 
features are used as inputs and ground truths are used as targets in MLP. The MLP is trained using Levenberg-Marquardt (LM) method [4]. After training, MLP classifier is used to classify the pixels of test MR images into either lesion or healthy tissues. Finally, connected component labelling algorithm is used in the post-processing step for making the detection more accurate.

\section{MATERIALS AND METHODS}

The proposed method is a semi-automatic and consists of denoising, intensity inhomgeneity correction, feature extraction, pixel classification using MLP classifier and extraction of lesion from segmented images using connected component labelling algorithm. The flowchart of the proposed method is given in Fig.1 Manually labelled ground truth is needed in supervised training of MLP using LM algorithm and user interaction is needed in the final stage while extracting the lesion from segmented images using connected component labelling algorithm. The proposed segmentation methodology has six steps. These steps are given below:

(1) data acquisition.

(2) Denoising using $3 \times 3$ median filter.

(3) Intensity inhomogeneity (IIH) correction

(4) Statistical feature extraction

(5) Segmentation using Multi-Layer Perceptron Neural Network.

(6) Extraction of lesions from segmented MR images.

The different steps of the proposed segmentation method are described in below:

\subsection{MRI Data Acquisition}

The proposed method is applied on two data sets. The MRI data sets used in this work have been collected from EKO-CT X-Ray, Medical College and Hospital, College Street, Kolkata-700073. First data set contains six Axial-T2 MR images and second data set contains six Sagittal-T2 MR images. All images are generated by 1.5-T GE Medical MRI imaging device. The thickness of slice is $5.0 \mathrm{~mm}$ and the slice gap is $1.5 \mathrm{~mm}$. The resolution of each MRI slice is $256 \times 256$.

\subsection{Denoising and IIH Correction}

The difficulties in proper segmentation as well as lesion detection occur due to the presence of noise and intensity inhomogeneity in MR image. The imperfections in image acquisition process results in intensity inhomogeneity in MR image. The noise across the MR images is removed using median filter with size $3 \times 3$ [9]. After denoising, the intensity inhomogeneity is corrected using Max filter based method [5, 11].

\subsection{Statistical Feature Extraction}

The four statistical features are extracted from denoised and intensity inhomogeneity corrected images. The statistical features [18] are calculated from each pixel's neighbourhood with size $N_{h}=3 \times 3$ as following:

(i) Mean: The mean defines the average level of intensity of the neighbourhood of $i^{\text {th }}$ pixel.

$$
\begin{gathered}
\mu_{i}=\sum_{j=1}^{N_{h}} j \cdot p(j) \\
p(j)=\frac{h(j)}{N_{h}}
\end{gathered}
$$

(ii) Standard Deviation:

$$
\sigma_{i}=\sqrt{\sum_{j=1}^{N_{h}}\left(j-\mu_{i}\right)^{2} \cdot p(j)}
$$

(iii) Skewness: The skewness defines the symmetry of an image.

$$
\mu_{i}^{3}=\sigma^{-3} \sum_{j=1}^{N_{h}}\left(j-\mu_{i}\right)^{3} \cdot p(j)
$$

(iv) Kurtosis: The kurtosis defines the measure of flatness of the histogram.

$$
\mu_{i}^{4}=\sigma^{-4} \sum_{j=1}^{N_{h}}\left(j-\mu_{i}\right)^{4} \cdot p(j)-3
$$

\subsection{Segmentation using MLP Classifier}

The gray levels and extracted features from the training images are used as inputs to the MLP classifier. The MLP has five input nodes in input layer, eleven hidden nodes in hidden layer and two output nodes in output layer. MLP is used as a binary classifier which classifies the pixels of image into either lesion or healthy tissues. The MLP is trained with LM algorithm. A MLP classifier is given in Fig. 2 .

The trained MLP is used to segment the test MR images into lesion and healthy tissues in brain. For each data set, one MR image is used for training and remaining five MR images are used in testing.

\subsection{Lesion Extraction}

In the segmented images few pixels from scalp and CSF are classified as lesions because they share similar intensity as that of the lesions. Finally, the connected component labelling algorithm [9] is used to separate the lesion from these healthy tissues in the segmented images.

\section{RESULTS AND ANALYSIS}

The performance of the proposed method is compared with Kmeans algorithm [19]. The K-means algorithm is applied on the gray levels of same denoised and intensity inhomogeneity corrected images. The results of K-means are generated by applying connected component labelling algorithm after thresholding the segmented images using highest centroid values.

In LM algorithm, the maximum number of epochs is 1000 and threshold error is set to 0.01 . initial regularizing parameter $\mu=$ $0.001, \mu$ decrease and increase factors are 0.1 and 10 respectively, maximum $\mu=1 e 10$. K-Means cluster algorithm is run with cluster 5 and maximum 5000 function evaluations.

The performance of the proposed method is measured both qualitatively as well as quantitatively. The qualitative performance is measured by visual analysis of the segmented image. The qualitative results of the proposed method and K-means based method with a single MRI slice from first data set are given in Fig. 3 and second data set are given in Fig. 4 By the visual inspection and analysis of the segmented images with lesions, it is clear that the proposed method detects lesions in MRI more accurately than K-means based segmentation method.

The quantitative performance is measured using confusion matrix [11]. The accuracy, sensitivity, specificity and False Positive Rate (FPR) are derived using confusion matrix. These measures are calculated with the help of ground truth of MR images. The confusion matrix is given in Table 1 The accuracy is calculated as follows: 
Communications on Applied Electronics (CAE) - ISSN : 2394 - 4714

Foundation of Computer Science FCS, New York, USA

Volume 4 - No. 5, February 2016 - www.caeaccess.org

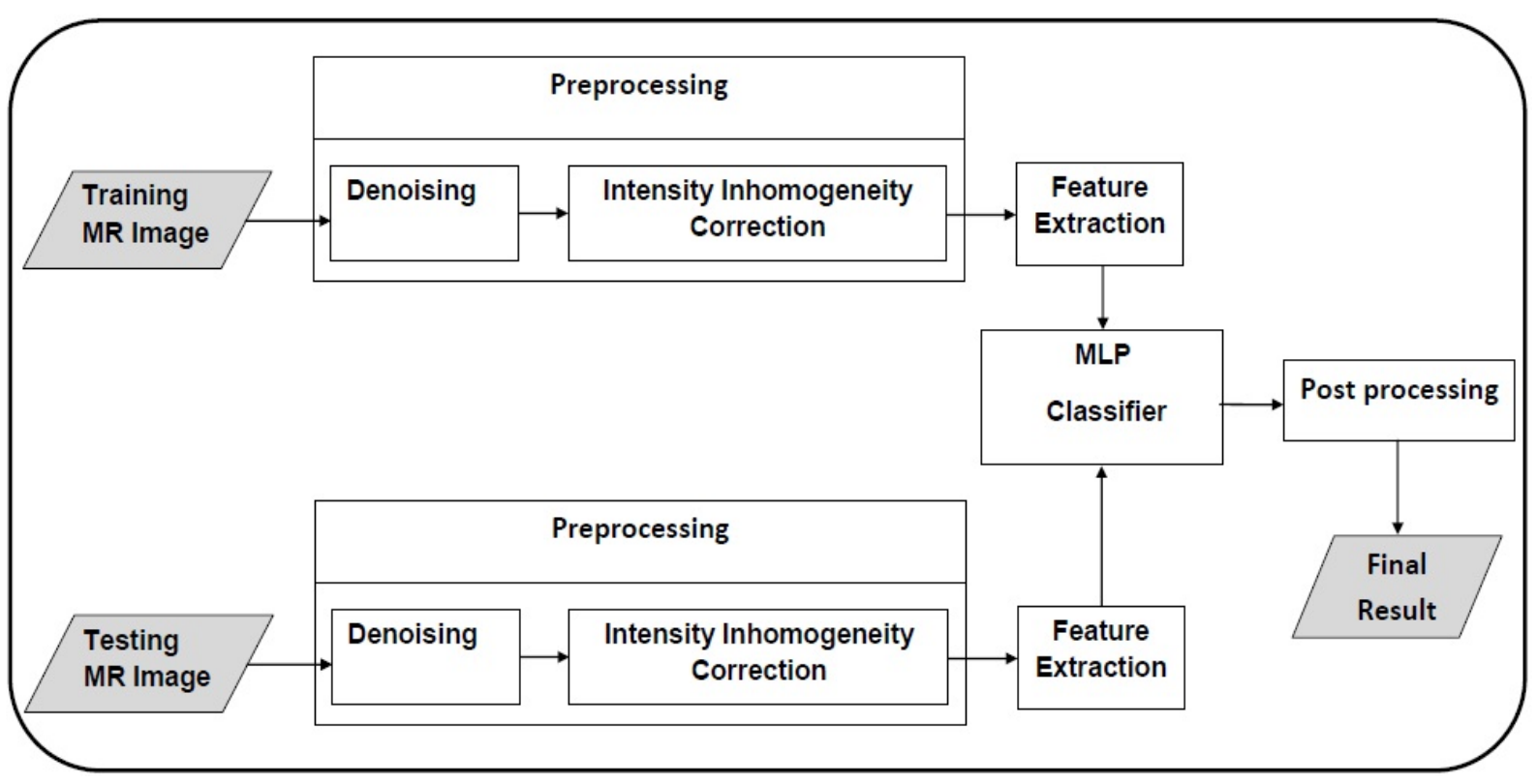

Fig. 1. Flowchart of the proposed method.

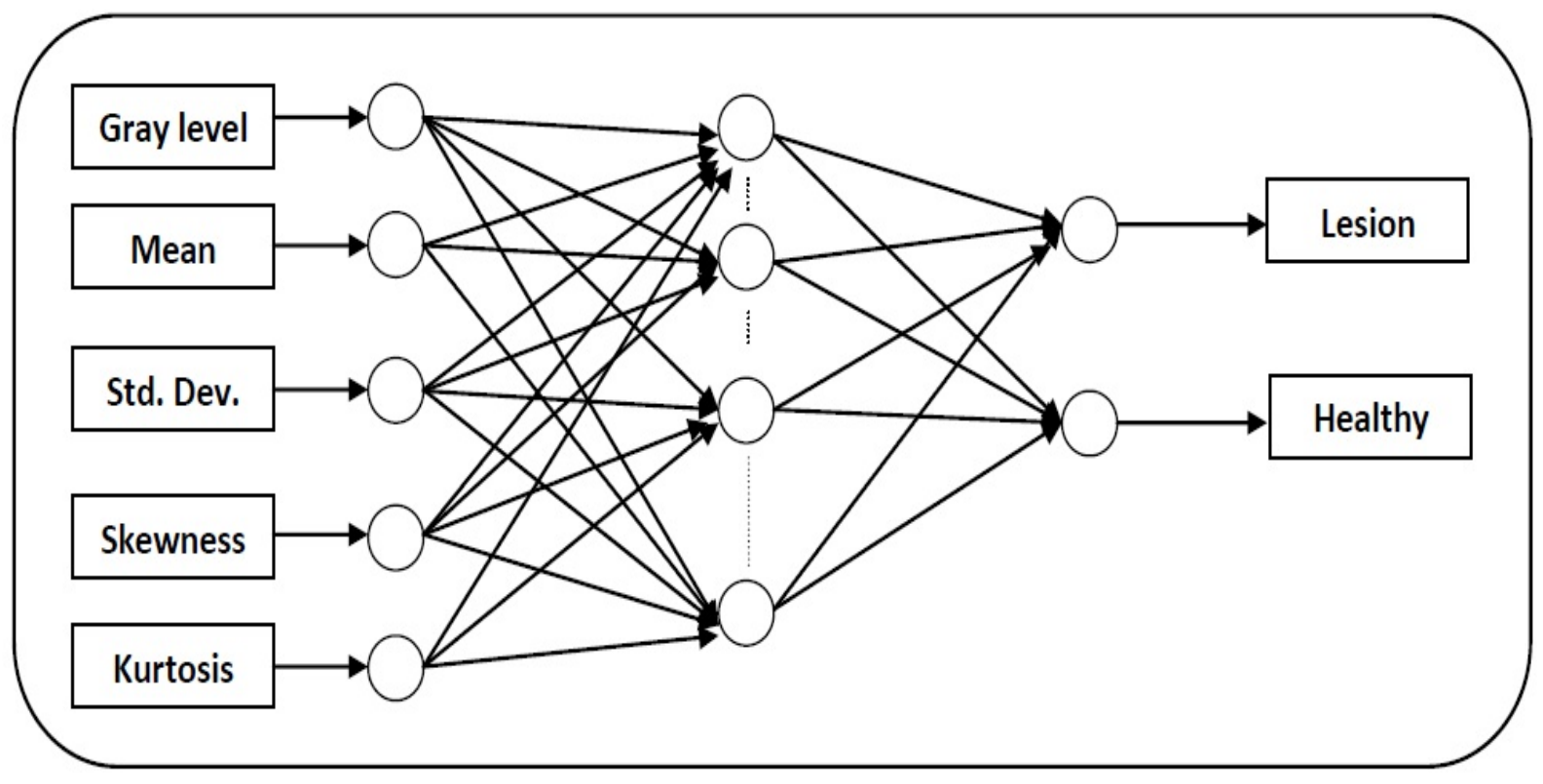

Fig. 2. MLP Classifier.

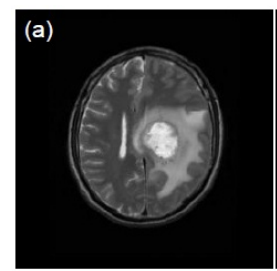

(b)
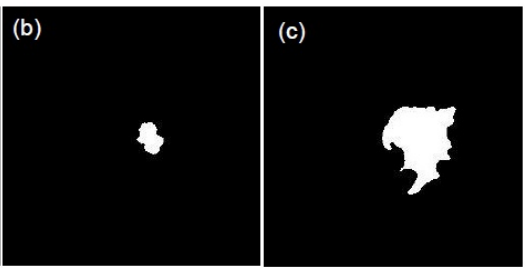

Fig. 3. (a) Original MRI slice in first data set, (b) segmented lesion using proposed method and (c) segmented lesion using K-means.

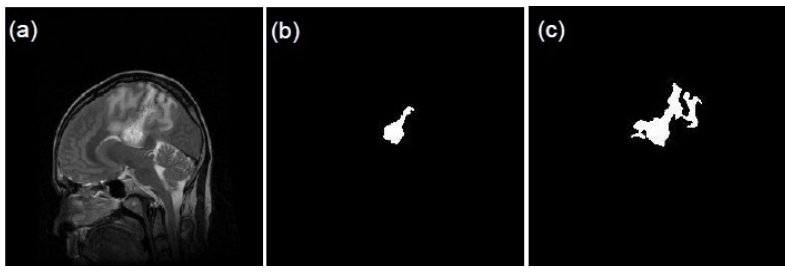

Fig. 4. (a) Original MRI slice in second data set, (b) segmented lesion using proposed method and (c) segmented lesion using K-means.

Sensitivity is the true positive rate and it is calculated as follows:

$$
\text { Accuracy }=\frac{T P+T N}{T P+F N+F P+T N} \times 100 \%
$$

$$
\text { Sensitivity }=\frac{T P}{T P+F N} \times 100 \%
$$


Table 1. Confusion Matrix

\begin{tabular}{|c|c|c|}
\hline Actual Class & \multicolumn{2}{|c|}{ Predicted Class } \\
\hline Positive & True Positives (TP) & False Negatives (FN) \\
\hline Negative & False Positives (FP) & True Negatives (TN) \\
\hline
\end{tabular}

and specificity is the true negative rate and it is calculated as follows:

$$
\text { Specitivity }=\frac{T N}{F P+T N} \times 100 \%
$$

FPR is calculated as follows:

$$
F P R=\frac{F P}{F P+T N} \times 100 \%
$$

Higher values of accuracy, sensitivity, specificity and lower FPR indicate better performance.

Dice similarity (DS) coefficient [20] is also used to evaluate the performance of the proposed method quantitatively. DS is calculated as following:

$$
D S(A, B)=\frac{2|A \cap B|}{|A| \cup|B|}
$$

where $A$ and $B$ are binary masks for the segmented lesion and ground truth. DS indicates overlapping ratio of detected lesion to the ground truth. The higher DS values indicate better performance. The mean and standard deviation of accuracy, sensitivity, specificity, FPR and DS for both data set 1 and data set 2 are given in Table 2 and Table 3 respectively. From the Table 2 and Table 3 , it is observed that the mean accuracy and specificity values are higher than that of K-means based method. FPR values of the proposed method are lower than that of K-means based method. The average sensitivity for K-means method is higher for both data sets because the detected lesion regions by K-means method cover the maximum of ground truths. Higher FPR values indicate that K-means method wrongly classify more healthier tissues as lesion which is undesirable. The DS values of the proposed method is much higher than that of K-means based method. This indicates that the overlapping of segmented lesions to the ground truths is more accurate than K-means based method. These quantitative measures signify that the proposed method performs better than K-means based method. The proposed method is more robust than K-means based method because the standard deviation of accuracy and DS values obtained from the proposed method are lower than that of K-means based method. The network size of the MLP is small and LM algorithm is very efficient for training. The convergence of $\mathrm{LM}$ algorithm is stable and fast. The above experimental results demonstrate that the proposed method is efficient and effective for lesion detection in brain MR images.

Table 2. Quantitative performance measures for

\begin{tabular}{|l|l|l|}
\multicolumn{3}{|c}{$1^{\text {st }}$ data set. } \\
\hline Measures & Proposed Method & K-Means \\
\hline Accuracy & $99.789 \pm 0.1160$ & $96.76 \pm 1.7628$ \\
\hline Sensitivity & $88.27 \pm 9.5140$ & $99.98 \pm 0.0500$ \\
\hline Specificity & $99.89 \pm 0.0858$ & $96.73 \pm 1.7802$ \\
\hline FPR & $0.11 \pm 0.08577$ & $3.27 \pm 1.7802$ \\
\hline DS & $0.87 \pm 0.0930$ & $0.42 \pm 0.2498$ \\
\hline
\end{tabular}

\section{CONCLUSION}

This paper proposes a novel lesion segmentation method in MRI of brain. A MLP is used to classify the pixels of MR images into lesion and healthy tissues. The MLP is trained using LevenbergMarquardt algorithm with one MRI slice from the data set and tested with remaining slices. The experimental results establishes that the proposed method performs better than K-means
Table 3. Quantitative performance measures for

\begin{tabular}{|l|l|l|}
\multicolumn{3}{|c}{$2^{\text {nd }}$ data set. } \\
\hline Measures & Proposed Method & K-Means \\
\hline Accuracy & $99.59 \pm 0.1752$ & $98.40 \pm 1.9913$ \\
\hline Sensitivity & $91.38 \pm 7.32$ & $96.72 \pm 3.9395$ \\
\hline Specificity & $99.71 \pm 0.2371$ & $98.45 \pm 2.0472$ \\
\hline FPR & $0.29 \pm 0.2371$ & $1.55 \pm 2.0472$ \\
\hline DS & $0.79 \pm 0.1629$ & $0.65 \pm 0.3681$ \\
\hline
\end{tabular}

both qualitatively and quantitatively. The proposed method can be further improved by using wavelet features instead of statistical features of MR images. The evolutionary neural systems can be used as classifier in the proposed methodology.

\section{REFERENCES}

[1] Tonarelli, L. 2013. Magnetic Resonance Imaging of Brain Tumor, CEwebsource.com.

[2] Parizel P. M., Hauwe L. V. D., Belder F. D., Goethem J. V., Venstermans C., Salgado R., Voormolen M., and Hecke W. V. 2010. Magnetic Resonance Imaging of the Brain, P. Reimer et al. (eds.): Clinical MR Imaging, Springer-Verlag Berlin Heidelberg

[3] Alirezaie J., Jernigan M.E. and Nahmias C. 1997. Neural Network based Segmentation of Magnetic Resonance Images of the Brain, IEEE Transactions on Nuclear Science, 44(3), 194-198

[4] Hyakin S. 2011. Neural Networks and Learning Machines, PHI, 3rd Eds.

[5] Balafar M.A., Ramli A.R. and Mashohor S. 2010. A new method for MR grayscale inhomogeneity correction, Artif. Intell. Rev., 34, 195-204.

[6] Wang S. and Summers R. M. 2012. Machine learning and radiology, Medical Image Analysis, 16, 933-951.

[7] El-Dahshan E. S. A., Mohsen H. M., Revett K. and Salem A. B. M. 2014. Computer-aided diagnosis of human brain tumor through MRI: A survey and a new algorithm, Expert Systems with Applications, 41, 5526-5545.

[8] Mayer A. and Greenspan H. 2009. An Adaptive Mean-Shift Framework for MRI Brain Segmentation, IEEE Trans. on Medical Imaging, Vol. 28, No. 8, 1238-1250.

[9] Si T., De A. and Bhattacharjee A.K. 2015. Grammatical Swarm based Segmentation Methodology for Lesion Segmentation in Brain MRI, International Journal of Computer Applications, 121(4), 1-8

[10] Si T., De A. and Bhattacharjee A.K. 2015. Brain's MRI Segmentation for Lesion Detection using Clustering with Grammatical Swarm Based-Adaptable Particle Swarm Optimizer, Journal of Network and Innovative Computing, MIR Labs, USA, Volume 3, 138-145.

[11] Si T., De A. and Bhattacharjee A.K. 2015. Brain MRI segmentation for tumor detection via entropy maximization using Grammatical Swarm, Int. J. Wavelets Multiresolut Inf. Process., 13(5), 1-8.

[12] Yang M.S., Lin K.C.R, Liu H.C. and Lirng J.F. 2007. Magnetic resonance imaging segmentation techniques using batch-type learning vector quantization algorithms, Magnetic Resonance Imaging, 25, Elsevier, 265-277

[13] Zhang N., Ruan S., Lebonvallet S., Liao Q. and Zhu Y. 2011. Kernel feature selection to fuse multi-spectral MRI images for brain tumor segmentation, Computer Vision and Image Understanding, 115, 256-269.

[14] Ortiz A., Gorriz J. M., Ramirez J., and Salas-Gonzalez D. 2012. Unsupervised Neural Techniques Applied to MR Brain Image Segmentation, Advances in Artificial Neural 
Systems, Hindawi Publishing Corporation, Volume 2012, Article ID 457590, 7 pages, doi:10.1155/2012/457590

[15] Song T., Jamshidi M.M., Lee R. R. and Huang M. 2007. A Modified Probabilistic Neural Network for Partial Volume Segmentation in Brain MR Image, IEEE Trans. On Neural Networks, Vol. 18, No. 5, 1424-1432.

[16] Zhang Y., Dong Z., Wua L. and Wanga S. 2011. A hybrid method for MRI brain image classification, Expert Systems with Applications, 38, pp. 10049-10053.

[17] Zhang Y., Wang S., Ji G. and Dong Z. 2013. An MR Brain Images Classifier System via Particle Swarm Optimization and Kernel Support Vector Machine, Hindawi Publishing Corporation The Scientific World Journal, Volume 2013, Article ID 130134, 9 pages, http://dx.doi.org/10.1155/2013/130134

[18] Selvaraj D. and Dhanasekaran R. 2013 A Review on Tissue Segmentation and Feature Extraction of MRI Brain images, International Journal of Computer Science \& Engineering Technology, 4(10), 1313-1332

[19] MacQueen J. 1967. Some methods for classification and analysis of multivariate observations, in Proc. 5th Berkeley Symp. Math. Stat. Probability, 281-297.

[20] Iftekharuddin K. M., Islam M. A., Shaik J., Parra C. and Ogg R. 2005. Automatic brain tumor detection in MRI: Methodology and statistical validation, SPIE Med. Imag., 5747, 2012-2022. 